\title{
Adaptive Cluster Synchronization for Nondelayed and Delayed Coupling Complex Networks with Nonidentical Nodes
}

\author{
Ze Tang and Jianwen Feng \\ College of Mathematics and Computational Sciences, Shenzhen University, Shenzhen, Guangdong 518060, China \\ Correspondence should be addressed to Jianwen Feng; fengjw@szu.edu.cn
}

Received 5 August 2012; Revised 21 November 2012; Accepted 18 December 2012

Academic Editor: Shukai Duan

Copyright (C) 2013 Z. Tang and J. Feng. This is an open access article distributed under the Creative Commons Attribution License, which permits unrestricted use, distribution, and reproduction in any medium, provided the original work is properly cited.

\begin{abstract}
We focus on the cluster synchronization problem for a kind of general networks with nondelayed and delayed coupling. Based on the pinning control scheme, a small fraction of the nodes in each cluster are pinned for successful control, and the states of the whole dynamical networks can be globally forced to the objective cluster states. Sufficient conditions are derived to guarantee the realization of the cluster synchronization pattern for all initial values by means of the Lyapunov stability theorem and linear matrix inequalities (LMIs). By using the adaptive update law, relative smaller control gains are obtained, and hence the control cost can be substantially lower. Numerical simulations are also exploited to demonstrate the effectiveness and validity of the main result.
\end{abstract}

\section{Introduction}

In the past few decades, synchronization and control problems are being widely studied [1-9]. Synchronization can be understood as the adjustment of rhythms or coherence of states by interaction [8] and realized via a sufficient information exchange among the nodes' interconnection in dynamical networks. The meaning of synchronization can interpret the essence of the collective behavior in nature and it has many potential applications in such areas as biological systems [1], secure communications [3], information processing, mechanical engineering, identification and pattern recognition [4], neuronal networks $[2,6,7,9]$, and so forth. In literature, there are many widely studied synchronization patterns, which define the correlated in-time behaviors among the nodes in a dynamical network, such as phase synchronization [3], impulsive synchronization [4], and complete synchronization [5].

Cluster synchronization has been well studied in recent works [10-14]. It requires that the coupled oscillators split into subgroups called clusters, such that the oscillators synchronized with one another in the same cluster, but there is no synchronization among different clusters. In [11], Wu et al. acquired a general network to a selected cluster synchronization pattern by means of a pinning control strategy. Wang et al. in [12] considered the dynamical networks with community structure and nonidentical nodes and with identical local dynamics for all individual nodes in each community, a way to design the controllers is very far-reaching, which motivates the present study. In [13], the cluster synchronization in an array of hybrid coupled neural networks with delay was investigated by Cao and Li. A method to realize cluster synchronization by constructing a special coupling matrix was proposed and several sufficient conditions for cluster synchronization based on the Lyapunov stability theory and the linear matrix inequality (LMI) technique were derived.

Some networks could achieve synchronization by utilizing information exchanges among local connections, while most networks in reality are generated randomly, which means they could not fulfill synchronization only depending on the coupling without using external forces. Hence, in many cases, negative state feedback control schemes are necessary to be designed to force the final synchronous state into a certain required objective trajectory. In the previous work [11], Wu et al. have proved that one can always pin a coupled complex network by adding some negative feedback controllers if the control strength is large enough. However, in practice, it is not allowed that the control gains are arbitrarily large. For synchronization, it was pointed out that theoretical values of the control gains are much larger 
than needed in practice. Therefore, one may ask: Can we find the sharp bound of the control gains? Similarly, in a pinning process, it is also important to make the control strength as small as possible. Some results about the adaptive adjustment control were presented in [15-19]. In [15], Lu and Cao proposed a simple scheme for the synchronization of an uncertain complex dynamical network with delayed coupling. The adaptive controllers to ensure the states of uncertain dynamical network with coupling delays to globally asymptotically synchronize were designed. A class of chaotic neural networks with known or unknown parameters by using the adaptive control and linear feedback with update law was investigated by He and Cao in [16]. They derived an adaptive feedback scheme to achieve synchronization of two coupled neural networks with a time-varying delay.

It is worth noticing that most of the studies on cluster synchronization for complex dynamical networks have been performed under some implicit assumptions that there exists the information communication of nodes via the edges only at time $t$ or at time $t-\tau$. However, in many circumstances, this simplification does not match satisfactorily the peculiarities of real networks: there exists the information communication of nodes not only at time $t$ or $t-\tau$ but also at time $t$, $t-\tau$. This general complex dynamical network model with time nondelayed and delayed coupling was investigated in $[14,20-23]$ and so forth. In [21], Xu and Su studied the general complex dynamical networks for synchronization and pinning control, they obtained less conservative criteria for both continuous-time and discrete-time complex dynamical networks with time delayed and nondelayed coupling by linearizing the system, and hence their results were established locally. Lou et al. discussed the adaptive synchronization of two complex networks with nondelayed and delayed couplings in [22]. They found that the coupling configuration matrices were not necessarily symmetric or irreducible and presented several criteria for synchronization of the two complex networks based on the Lyapunov stability theory. The exponential cluster synchronization of coupled impulsive genetic oscillators with external disturbances and communication delay was investigated by Zhang et al. in [14]. Some cluster synchronization criteria for the delayed and nondelayed coupling impulsive genetic oscillators were presented and the maximal allowable bound of time delay was obtained as well. However, the cluster synchronization problems for the nondelayed and delayed coupling networks in [14] were established based on the strong assumption that all of the nodes have the same dynamical behaviors.

In this paper, we study the cluster synchronization problem for a kind of general complex dynamical networks with time nondelayed and delayed coupling. We emphasize that the dynamical behaviors of the nodes in different clusters are distinct too. Some global synchronization criteria are derived by means of the pinning control method. The adaptive adjustment controllers are designed skillfully as they have the potential ability to obtain more suitable control gains, which are more important in reality. Moreover, we apply the Lyapunov stability theorem to ensure the realization of cluster synchronization. The required global synchronization criteria then follows immediately from the LMIs.
The rest of the paper is organized as follows. In Section 2, we present some preparations and the general complex dynamical network model. In Section 3, we analyze the cluster synchronization of the network with time nondelayed and delayed coupling under the adaptive control. Numerical simulations are given to verify our theoretical results in Section 4. Concluding remarks are drawn in Section 5.

Notations. The mark $A^{T}$ denotes the transport of the matrix $A$. $R^{n}$ denotes the $n$-dimensional Euclidean space. $R^{n \times n}$ are $n \times n$ real matrices. diag $\{\cdots\}$ stands for a diagonal matrix. The sign $\|\cdot\|$ stands for the Euclid norm of the matrix or the vector. A symmetric real matrix $A$ is positive definite (semidefinite) if $x^{T} A x>0(\geq 0)$ for all nonzero $x$ we denote this as $A>0(A \geq$ $0) . I$ stands for the identity matrix with proper dimension. The dimension of these vectors and matrices will be cleared in the context.

\section{Preliminaries and Model Description}

We first make some assumptions for a network with cluster structure as follows. Suppose that these networks have $N$ nodes and $m$ clusters with $N>m \geq 2$. Let $\mu_{i}=j$ if node $i$ belongs to the $j$ th cluster. Denote $U_{j}$ as the set of all nodes in the $j$ th cluster and $\widehat{U}_{j}$ as the set consisting of all nodes in the $j$ th cluster which have direct connections to the nodes in other clusters. Thus we have (1) $U_{i} \cap U_{j}=\phi$, for $i \neq j$ and $i, j=1,2, \ldots, m ;(2) \bigcup_{i=1}^{m} U_{i}=\{1,2, \ldots, N\}$.

Some preliminaries such as definitions and lemmas will be given in the following, which are necessary throughout the paper.

Definition 1 (see [24]). Synchronization manifold

$$
M=\left\{\left(x_{1}^{T}, \ldots, x_{N}^{T}\right)^{T} \in R^{N \times n}: x_{i}=x_{j}, i, j=1,2, \ldots, N\right\},
$$

where $x_{i}=\left(x_{i 1}, \ldots, x_{i n}\right)^{T} \in R^{n}$ for $i=1,2, \ldots, N$.

Definition 2 (see [12]). A network with $N$ oscillators is said to achieve cluster synchronization if it satisfies

$$
\lim _{t \rightarrow+\infty}\left\|x_{i}(t)-s_{\mu_{i}}(t)\right\|=0, \quad i=1,2, \ldots, N
$$

where $s_{\mu_{i}}(t) \in R^{n}$ is a solution of an isolate node and satisfies

$$
\dot{s}_{\mu_{i}}(t)=f_{\mu_{i}}\left(s_{\mu_{i}}(t)\right), \quad i=1,2, \ldots, N
$$

which describes the identical local dynamics for the nodes in the $\mu_{i}$ th cluster (community).

Definition 3 (see [11]). Function class $\operatorname{QUAD}(\Delta, P, \eta)$ : let $\Delta=$ $\operatorname{diag}\left\{\delta_{1}, \ldots, \delta_{n}\right\}$ be a diagonal matrix and $P=\operatorname{diag}\left\{p_{1}, \ldots\right.$, $\left.p_{n}\right\}$ a positive-definite diagonal matrix. $\operatorname{QUAD}(\Delta, P, \eta)$ 
denotes a class of continuous functions $f(x, t): R^{n} \times$ $[0,+\infty) \rightarrow R^{n}$ satisfying

$$
\begin{gathered}
(x-y)^{T} P(f(x, t)-f(y, t)-\Delta(x-y)) \\
\leq-\eta(x-y)^{T}(x-y)
\end{gathered}
$$

for some $\eta>0$, all $x, y \in R^{n}$ and all $t \geq 0$.

Lemma 4 (Schur complement [25]). The following linear matrix inequality (LMI)

$$
\left(\begin{array}{ll}
Q(x) & S(x) \\
S(x)^{T} & R(x)
\end{array}\right)>0
$$

is equivalent to one of the following conditions:

(1) $Q(x)>0, R(x)-S(x)^{T} Q(x)^{-1} S(x)>0$;

(2) $R(x)>0, Q(x)-S(x) R(x)^{-1} S(x)^{T}>0$,

where $Q(x)$ and $R(x)$ are symmetric matrices and $S(x)$ is a matrix with suitable dimensions.

In general, the $\mu_{i}$ th cluster equation with time nondelayed and delayed coupling under pinning control scheme $u_{i}(t)$ can be described as

$$
\begin{array}{r}
\dot{x}_{i}(t)=f_{\mu_{i}}\left(x_{i}(t)\right)+\sum_{j=1}^{N} a_{i j} \Gamma x_{j}(t)+\sum_{j=1}^{N} b_{i j} \Gamma x_{j}(t-\tau)+u_{i}(t), \\
i=1, \ldots, N,
\end{array}
$$

where $N$ is the number of nodes and $x_{i}(t)=\left(x_{i 1}, \ldots, x_{i n}\right)^{T} \epsilon$ $R^{n}$ are the state variables of the $i$ th node, $t \in[0,+\infty)$. $f_{\mu_{i}}: R^{n} \rightarrow R^{n}$ is a continuously differentiable function describes the local dynamics of nodes in the $\mu_{i}$ th cluster. For any pair of indices $i$ and $j$, if node $i$ and node $j$ belong to different clusters, that is, $\mu_{i} \neq \mu_{j}$, then $f_{\mu_{i}} \neq f_{\mu_{j}} . \Gamma=$ $\operatorname{diag}\left\{r_{1}, r_{2}, \ldots, r_{n}\right\} \in R^{n \times n}$ is the inner-coupling matrix with $r_{i} \geq 0, i=1,2, \ldots, n . A=\left(a_{i j}\right)$ and $B=\left(b_{i j}\right) \in$ $R^{N \times N}$ are the coupling matrices with zero-sum rows $a_{i i}=$ $-\sum_{j \neq i, j=1}^{N} a_{i j}, b_{i i}=-\sum_{j \neq i, j=1}^{N} b_{i j}, a_{i j}>0, b_{i j}>0$ for $i, j=1, \ldots, N$, which are determined by the topological structure of the networks. $u_{i}(t)(i=1,2, \ldots, N)$ are some local negative feedback controllers to be designed later. And we assume that $A$ and $B$ are symmetric and irreducible matrices. That means there is no isolate node in the system. This diffusivity condition implies that the coupling dynamical network would be decoupled when the array of nodes is synchronized.

\section{The Stability Analysis for Cluster Synchronization under Pinning Adaptive Control}

In this section, the cluster synchronization problem amounts to proving the stability of the error system. By using a suitably chosen control scheme, that is, pinning adaptive controllers that control only a fraction of the nodes in every cluster which have direct connections with the nodes in other clusters will be designed to globally stabilize the dynamical network with nondelayed and delayed coupling. We present the main result of this paper in the form of Theorem 6 , then give the detailed proof process.

Let

$$
e_{i}(t)=x_{i}(t)-s_{\mu_{i}}(t)
$$

be the error state of node $i$ between the current state $x_{i}(t)$ and the cluster objective state $s_{\mu_{i}}(t)$, where $s_{\mu_{i}}(t)$ are defined as in Definition 2 for $i=1, \ldots, N$; they are the cluster objective trajectories that the dynamical network (6) will be forced to.

Transform the system (6) yields the following error dynamical system:

$$
\begin{aligned}
\dot{e}_{i}(t)= & f_{\mu_{i}}\left(x_{i}(t)\right)-f_{\mu_{i}}\left(s_{\mu_{i}}(t)\right)+\sum_{j=1}^{N} a_{i j} \Gamma e_{j}(t) \\
& +\sum_{j=1}^{N} b_{i j} e_{j}(t-\tau)+u_{i}(t) \\
& +\sum_{j=1}^{N} a_{i j} \Gamma s_{\mu_{i}}(t)+\sum_{j=1}^{N} b_{i j} \Gamma s_{\mu_{i}}(t-\tau), \quad i=1,2, \ldots, N .
\end{aligned}
$$

According to the zero-row-sum condition, it is not difficult to get

$$
\sum_{j=i}^{N} a_{i j} \Gamma s_{\mu_{i}}(t)=0, \quad \sum_{j=i}^{N} b_{i j} \Gamma s_{\mu_{i}}(t-\tau)=0
$$

if and only if $i \in U_{\mu_{i}} \backslash \widehat{U}_{\mu_{i}}$ for $i=1,2, \ldots, N$.

In view of this special property, we design the local linear negative feedback controllers as

$$
u_{i}(t)= \begin{cases}-d_{i}(t) e_{i}(t)-\sum_{j=1}^{N} a_{i j} \Gamma s_{\mu_{i}}(t) & \\ -\sum_{j=1}^{N} b_{i j} \Gamma s_{\mu_{i}}(t-\tau), & i \in \widehat{U}_{\mu_{i}}, \\ 0, & i \in U_{\mu_{i}} \backslash \widehat{U}_{\mu_{i}},\end{cases}
$$

where $d_{i}(t)>0, i=1,2, \ldots, N$, are the time-varying adaptive control gains that can be suitably chosen by the cluster synchronization system (6). To guarantee negative feedback, the adaptive gains are designed as

$$
\dot{d}_{i}(t)=\alpha_{i} e_{i}(t)^{T} P e_{i}(t), \quad i=1,2, \ldots, N
$$

where $\alpha_{i}, i=1,2, \ldots, N$, are positive constants to be determined. 
Remark 5. Through the above controllers, the first term in (10) is used to synchronize all nodes in the same cluster, while the remainder term in the controller is to weaken the mutual influences among clusters at the intersection nodes. By the way, we design the adaptive gains as (11) (adaptive update law) in order to make the control strength as small as possible, and hence the control cost can be substantially lower and our strategy can be easier to implement.

In the following, the main result of cluster synchronization criteria for nondelayed and delayed coupling under the pinning adaptive control scheme (10) and the adaptive update law (11) will be given.

Theorem 6. Suppose $f_{\mu_{i}}(\cdot) \in \operatorname{QUAD}(P, \Delta, \eta), i=1, \ldots, N$, such that

$$
\left[\begin{array}{cc}
r_{k} A+\delta_{k} I-D+Q & \frac{1}{2} r_{k} B \\
\frac{1}{2} r_{k} B & -Q
\end{array}\right]<0
$$

hold for all $k=1,2, \ldots, n$, then the cluster synchronization manifold

$$
M=\left(s_{\mu_{1}}, \ldots, s_{\mu_{N}}\right)
$$

of the network (6) is globally stable for any time delay $\tau>0$ and for any initial values under the pinning state feedback controllers (10) and the adaptive update law (11), where $Q \in R^{n \times n}$ is a positive definite diagonal matrix and $D=$ $\operatorname{diag}\left\{d_{1}, d_{2}, \ldots, d_{N}\right\}$.

Proof. For notational convenience, we denote

$$
\begin{gathered}
\widetilde{e}_{i}(t)=\left(e_{1 i}(t), \ldots, e_{N i}(t)\right)^{T}, \\
\widetilde{e}_{i}(t-\tau)=\left(e_{1 i}(t-\tau), \ldots, e_{N i}(t-\tau)\right)^{T} .
\end{gathered}
$$

Select the following Lyapunov functional candidate as

$$
\begin{aligned}
V(t)= & \frac{1}{2} \sum_{i=1}^{N} e_{i}(t)^{T} P e_{i}(t)+\sum_{i=1}^{N} \frac{1}{2 \alpha_{i}}\left(d_{i}(t)-d_{i}\right)^{2} \\
& +\sum_{k=1}^{n} p_{k} \int_{t-\tau}^{t} \widetilde{e}_{k}(s)^{T} Q \widetilde{e}_{k}(s) d s
\end{aligned}
$$

where $d_{i}, i=1,2, \ldots, N$, are positive constants to be determined. The derivative of $V(t)$ along the trajectories of the error system (8) with considering the pinning adaptive control scheme (10) and the adaptive update law (11) as described above is

$$
\begin{aligned}
& \dot{V}(t)=\sum_{i=1}^{N} e_{i}(t)^{T} P \dot{e}_{i}(t)+\sum_{i=1}^{N} \frac{1}{\alpha_{i}}\left(d_{i}(t)-d_{i}\right) \dot{d}_{i}(t) \\
& +\sum_{k=1}^{n} p_{k}\left[\widetilde{e}_{k}(t)^{T} Q \widetilde{e}_{k}(t)-\widetilde{e}_{k}(t-\tau)^{T} Q \widetilde{e}_{k}(t-\tau)\right] \\
& =\sum_{i=1}^{N} e_{i}(t)^{T} P\left[f_{\mu_{i}}\left(x_{i}(t)\right)-f_{\mu_{i}}\left(s_{\mu_{i}}(t)\right)+\sum_{j=1}^{N} a_{i j} \Gamma e_{j}(t)\right. \\
& +\sum_{j=1}^{N} b_{i j} \Gamma e_{j}(t-\tau)+\sum_{j=1}^{N} a_{i j} \Gamma s_{\mu_{i}}(t) \\
& \left.+\sum_{j=1}^{N} b_{i j} \Gamma s_{\mu_{i}}(t-\tau)+u_{i}(t)\right] \\
& +\sum_{i=1}^{N} \frac{1}{\alpha_{i}}\left(d_{i}(t)-d_{i}\right) \alpha_{i} e_{i}(t)^{T} P e_{i}(t) \\
& +\sum_{k=1}^{n} p_{k}\left[\widetilde{e}_{k}(t)^{T} Q \widetilde{e}_{k}(t)-\widetilde{e}_{k}(t-\tau)^{T} Q \widetilde{e}_{k}(t-\tau)\right] \\
& =\sum_{i=1}^{N} e_{i}(t)^{T} P\left[f_{\mu_{i}}\left(x_{i}(t)\right)-f_{\mu_{i}}\left(s_{\mu_{i}}(t)\right)-\Delta e_{i}(t)\right] \\
& +\sum_{i=1}^{N} e_{i}(t)^{T} P\left[\Delta e_{i}(t)+\sum_{j=1}^{N} a_{i j} \Gamma e_{j}(t)\right. \\
& \left.+\sum_{j=1}^{N} b_{i j} \Gamma e_{j}(t-\tau)-d_{i}(t) e_{i}(t)\right] \\
& +\sum_{i=1}^{N} e_{i}(t)^{T}\left(d_{i}(t)-d_{i}\right) P e_{i}(t) \\
& +\sum_{k=1}^{n} p_{k}\left[\widetilde{e}_{k}(t)^{T} Q \widetilde{e}_{k}(t)-\widetilde{e}_{k}(t-\tau)^{T} Q \widetilde{e}_{k}(t-\tau)\right] .
\end{aligned}
$$

According to Definition 3, we have

$$
\begin{aligned}
\dot{V}(t) \leq & -\eta \sum_{i=1}^{N} e_{i}(t)^{T} e_{i}(t) \\
& +\sum_{k=1}^{n} p_{k} r_{k}\left[\widetilde{e}_{k}(t)^{T} A \widetilde{e}_{k}(t)+\widetilde{e}_{k}(t)^{T} B \widetilde{e}_{k}(t-\tau)\right] \\
& +\sum_{k=1}^{n} p_{k} \widetilde{e}_{k}(t)^{T}\left(\delta_{k} I-D\right) \widetilde{e}_{k}(t)+\sum_{k=1}^{n} p_{k} \widetilde{e}_{k}(t)^{T} Q \widetilde{e}_{k}(t)
\end{aligned}
$$




$$
\begin{aligned}
& -\sum_{k=1}^{n} p_{k} \widetilde{e}_{k}(t-\tau)^{T} Q \widetilde{e}_{k}(t-\tau) \\
= & -\eta \sum_{i=1}^{N} e_{i}(t)^{T} e_{i}(t) \\
& +\sum_{k=1}^{n} p_{k} \widetilde{e}_{k}(t)^{T}\left[r_{k} A+\delta_{k} I-D+Q\right] \widetilde{e}_{k}(t) \\
& +\sum_{k=1}^{n} p_{k} \widetilde{e}_{k}(t)^{T}\left(r_{k} B\right) \widetilde{e}_{k}(t-\tau) \\
& -\sum_{k=1}^{n} p_{k} \widetilde{e}_{k}(t-\tau)^{T} Q \widetilde{e}_{k}(t-\tau) .
\end{aligned}
$$

With an eye to Lemma 4, we could know that if the condition (12) hold for $k=1, \ldots, n$, then we can get that

$$
\dot{V}(t) \leq-\eta \sum_{i=1}^{N} e_{i}(t)^{T} e_{i}(t)
$$

According to the Lyapunov stability theorem, one has

$$
e_{i}(t) \longrightarrow 0 \quad(t \longrightarrow+\infty)
$$

whcih equals to

$$
\lim _{t \rightarrow+\infty}\left\|e_{i}(t)\right\|=\lim _{t \rightarrow+\infty}\left\|x_{i}(t)-s_{\mu_{i}}(t)\right\|=0, \quad \forall i=1,2, \ldots, N .
$$

Combine with the designing of the adaptive controllers. It can be derived that

$$
\dot{d}_{i}(t) \longrightarrow 0 \quad(t \longrightarrow+\infty) \Longleftrightarrow d_{i}(t) \longrightarrow d_{i} \quad(t \longrightarrow+\infty),
$$

where $d_{i}(i=1, \ldots, N)$ are positive constants, it means that the nodes within $\mu_{i}$ th cluster are fully synchronized to dynamics state $s_{\mu_{i}}$, while nodes in separate clusters behave independently. Therefore, we get the global stability of the cluster synchronization manifold

$$
M=\left(s_{\mu_{1}}, \ldots, s_{\mu_{N}}\right) .
$$

That completes the proof of the main result.

Remark 7. The main theory explicitly showed a kind of effective and relatively cheap control strategy control scheme designed in this paper for the cluster synchronization of complex dynamical networks. That is, throughout the above proof, we can get some relative proper and practical control gains by using the pinning control scheme (10) as well as the adaptive control gains update law (11). Therefore, we can save much resources and costs for the smaller control gains than the previous works got in $[11,12]$. Although one can pin a coupled complex network by adding some negative feedback controllers if the control strength is large enough, it is not practical that the control gains are arbitrarily large.
In the following, we give two corollaries, which are the investigation of simplified complex networks (6). Those results could come to the conclusion that our main result in this paper is more comprehensive and meaningful than the previous ones.

Corollary 8. Suppose $f_{\mu_{i}}(\cdot) \in Q U A D(P, \Delta, \eta), i=1, \ldots, N$, such that

$$
\delta_{k} I+r_{k} A-D<0
$$

hold for $k=1,2, \ldots, n$, then the cluster synchronization manifold

$$
M=\left(s_{\mu_{1}}, \ldots, s_{\mu_{N}}\right)
$$

of the following dynamical complex network

$$
\dot{x}_{i}(t)=f_{\mu_{i}}\left(x_{i}(t)\right)+\sum_{j=1}^{N} a_{i j} \Gamma x_{j}(t)+u_{i}(t), \quad i=1, \ldots, N
$$

is globally stable for all initial values under the pinning state feedback control scheme

$$
u_{i}(t)= \begin{cases}-d_{i}(t) e_{i}(t)-\sum_{j=1}^{N} a_{i j} \Gamma s_{\mu_{i}}(t), & i \in \widehat{U}_{\mu_{i}}, \\ 0, & i \in U_{\mu_{i}} \backslash \widehat{U}_{\mu_{i}}\end{cases}
$$

and the adaptive update law (11), where $D=\operatorname{diag}\left\{d_{1}, d_{2}\right.$, $\left.\ldots, d_{N}\right\}$.

Remark 9. The similar proof process of Corollary 8 could be thought of as the simplified process of Theorem 6. Similar conclusion also can be found in [12]. In this special case, we investigate the complex network without time delay.

Corollary 10. Suppose $f_{\mu_{i}}(\cdot) \in Q U A D(P, \Delta, \eta), i=1, \ldots, N$, such that

$$
\left[\begin{array}{cc}
\delta_{k} I-D+Q & \frac{1}{2} r_{k} B \\
\frac{1}{2} r_{k} B & -Q
\end{array}\right]<0
$$

hold for $k=1,2, \ldots, n$, then the cluster synchronization manifold

$$
M=\left(s_{\mu_{1}}, \ldots, s_{\mu_{N}}\right)
$$

of the time delayed coupling dynamical network

$$
\dot{x}_{i}(t)=f_{\mu_{i}}\left(x_{i}(t)\right)+\sum_{j=1}^{N} b_{i j} \Gamma x_{j}(t-\tau)+u_{i}(t), \quad i=1, \ldots, N
$$

under the pinning control scheme

$$
u_{i}(t)= \begin{cases}-d_{i}(t) e_{i}(t)-\sum_{j=1}^{N} b_{i j} \Gamma s_{\mu_{i}}(t-\tau), & i \in \widehat{U}_{\mu_{i}}, \\ 0, & i \in U_{\mu_{i}} \backslash \widehat{U}_{\mu_{i}}\end{cases}
$$


and the adaptive update law (11) is globally stable for any time delay $\tau>0$, where $Q \in R^{n \times n}$ is a positive definite diagonal matrix and $D=\operatorname{diag}\left\{d_{1}, d_{2}, \ldots, d_{N}\right\}$.

Remark 11. The proof of Corollary 10 could be thought of as another simplification of the proof of Theorem 6; this corollary, of course, is another special situation of the main result, that is, the network with time delayed coupling. This corollary can tells the result in this paper including the previous works, so we did a more comprehensive work.

\section{Numerical Simulation}

The above criteria can be applied to networks with different topologies and sizes. In this section, we put an effective example to illustrate the validity of the theory given in previous sections.

Example 12. We use a chaotic Lorenz oscillator and a chaotic Chua's oscillator with different parameters, respectively, as the example to illustrate the main results (Theorem 6) of our paper. The chaotic Lorenz oscillator model could be described as follows:

$$
\left[\begin{array}{c}
\dot{x}_{1} \\
\dot{x}_{2} \\
\dot{x}_{3}
\end{array}\right]=\left[\begin{array}{c}
10\left(x_{2}-x_{1}\right) \\
28 x_{1}-x_{2}-x_{1} x_{3} \\
x_{1} x_{2}-\frac{8}{3} x_{3}
\end{array}\right],
$$

$$
A=[-1,1,0,0,0,0 ; 0,-1,1,0,0,0 ; 1,0,-1,0,0,0 ; 0,0,1,-2,1,0 ; 0,0,0,0,-1,1 ; 0,0,0,1,0,-1] \text {, }
$$

and the time delayed coupling matrix we assume is $B=0.5 A$; both of the matrices satisfy the diffusive condition. Cluster $U_{1}=\{1,2,3\}$ follows the dynamics of (30) while cluster $U_{2}=$ $\{4,5,6\}$ follows the dynamics of (31). We add the adaptive adjustment controllers on node 3 and 4 as they have direct connection between two clusters. derive

By using MATLAB LMI TOOLBOX on condition (11), we

$$
Q=\operatorname{diag}(0.1963,0.4452,0.7425,0.2687) \text {, }
$$

where $Q$ is a positive definite diagonal matrix which meets the requirement in Theorem 6.

Now define the error $E$ of cluster synchronization for this controlled network as

$$
E(t)=\sqrt{\frac{1}{l} \sum_{i=1}^{l}\left\|x_{i}(t)-s_{\mu_{i}}(t)\right\|,}
$$

where $l$ is the number of nodes in each cluster. Obviously, here $l=3$. Define

$$
E_{12}(t)=\left\|x_{i_{0}}(t)-x_{j_{0}}(t)\right\|, \quad i_{0} \in U_{1}, j_{0} \in U_{2}
$$

as the error between two communities. and the chaotic Chua's oscillator model is

$$
\left[\begin{array}{c}
\dot{x}_{1} \\
\dot{x}_{2} \\
\dot{x}_{3}
\end{array}\right]=\left[\begin{array}{c}
15\left(x_{2}-x_{1}+g\left(x_{1}\right)\right) \\
x_{1}-x_{2}+x_{3} \\
-25.5 x_{2}
\end{array}\right],
$$

where

$$
g(y)=\frac{5}{7} y+\frac{3}{14}(|y+1|-|y-1|)
$$

Their vector field functions are denoted by $f_{1}, f_{2}$, and $f_{3}$, respectively. According to Theorem 6, the validity of the result must satisfy the QUAD condition, so we should take the first step to verify Definition 3 to guarantee cluster synchronization for the network (6) under the pinning controller (10). To achieve the purpose, Wang et al. did a good job. The detailed proof process could be found in [12]; based on that paper analysis, we can set

$$
P=I_{3}, \quad \Delta=52 I_{3}, \quad \Gamma=I_{3} .
$$

The network consists of 6 nodes that are divided into two clusters $U_{1}=\{1,2,3\}$ and $U_{2}=\{4,5,6\}$ (see Figure 1(a)). From the network structure shown in Figure 1, we can get that the time nondelayed coupling matrix $A$ is

According to the conditions in Theorem 6, we know that the cluster synchronization manifold $M$ is globally stable for any positive time delay $\tau$. We plot the curves of the cluster synchronization errors of each cluster in Figure 1 (Figures 1(b) and 1(c) stand for cluster synchronization errors in cluster 1 and 2, resp., and Figure 1(d) represents for the error between two communities). It is clear that the cluster synchronization is achieved if $E(t)$ converges to zero and $E_{12}(t)$ do not as $t \rightarrow+\infty$. The pinning adaptive controllers give a successful realization of cluster synchronization with the varying control gain $d(t)$; in this situation, we derive the control gain matrix $D=24 I$. It is more practical in real applications than we got in the view of theories.

\section{Conclusion}

This paper considered a class of complex dynamical networks with time nondelayed and delayed coupling. Instead of controlling all nodes, pinning adaptive control was used to pin a small fraction of the nodes in each cluster for successfully control, and the states of the whole dynamical networks can be globally exponentially forced to the objective cluster states. We have derived sufficient criteria for cluster synchronization 


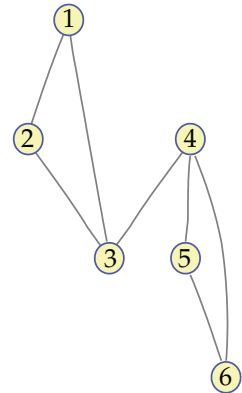

(a)

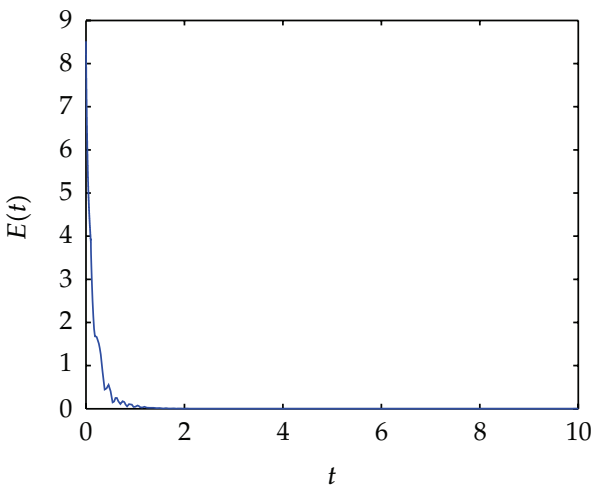

(c)

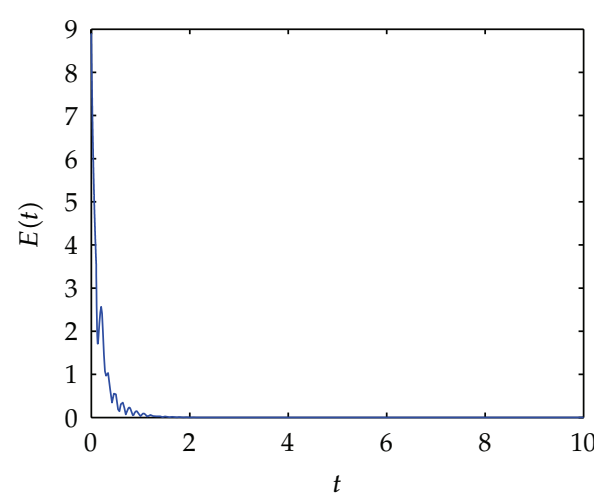

(b)

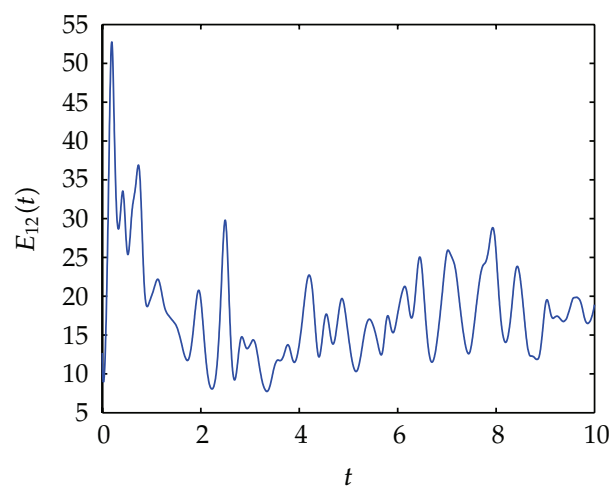

(d)

FIGURE 1: A network with size $N=6$ and two clusters shown in (a). (b, c) stand for the cluster synchronization error $E(t)$ in each cluster and (d) represents the error $E_{12}(t)$ between the two communities.

under pinning control as the situation of dynamics are different in every cluster, which is more practical and meaningful in realistic. The adaptive method to adjust the control gains suitably rather than as large as possible was introduced and global synchronization of the general complex networks were achieved by using the Lyapunov stability theorem and linear matrix inequality. The numerical simulation was exploited to demonstrate the effectiveness of the pinning adaptive strategy proposed in this paper.

\section{Acknowledgments}

The authors thank the referees and the editors for their valuable comments on this paper. The work is supported by the Natural Science Foundation of China (Grant no. 61273220), Guangdong Education University Industry Cooperation Projects (Grant no. 2009B090300355), and the Shenzhen Basic Research Project (JC201006010743A, JCYJ20120613105730482).

\section{References}

[1] S. H. Strogatz and I. Stewart, "Coupled oscillators and biological synchronization," Scientific American, vol. 269, no. 6, pp. 102109, 1993.
[2] C. M. Gray, "Synchronous oscillations in neural systems," Journal of Computational Neuroscience, vol. 1, no. 1-2, pp. 11-38, 1994.

[3] J. Fell and N. Axmacher, "The role of phase synchronization in memory processes," Nature Reviews Neuroscience, vol. 12, no. 2, pp. 105-118, 2011.

[4] W. He, F. Qian, J. Cao, and Q. L. Han, "Impulsive synchronization of two nonidentical chaotic systems with time-varying delay," Physics Letters A, vol. 375, no. 3, pp. 498-504, 2011.

[5] L. M. Pecora and T. L. Carroll, "Synchronization in chaotic systems," Physical Review Letters, vol. 64, no. 8, pp. 821-824, 1990.

[6] H. Y. Li, B. Chen, Q. Zhou, and W. Y. Qian, "Robust stability for uncertain delayed fuzzy Hopfield neural networks with Markovian jumping parameters," IEEE Transactions on Systems, Man, and Cybernetics B, vol. 39, no. 1, pp. 94-102, 2009.

[7] H. Y. Li, H. J. Gao, and P. Shi, "New passivity analysis for neural networks with discrete and distributed delays," IEEE Transactions on Neural Networks, vol. 21, no. 11, pp. 1842-1847, 2010.

[8] A. Pikovsky, M. Rosenblum, and J. Kurths, Synchronization: A Universal Concept in Nonlinear Sciences, vol. 12 of Cambridge Nonlinear Science Series, Cambridge University Press, Cambridge, UK, 2001.

[9] Y. Tang, H. Gao, W. Zou, and J. Kurths, "Identifying controlling nodes in neuronal networks in different scales," PLoS ONE, vol. 7, Article ID e41375, 2012. 
[10] J. Y. Wang, J. W. Feng, C. Xu, and Y. Zhao, "Cluster synchronization of nonlinearly-coupled complex networks with nonidentical nodes and asymmetrical coupling matrix," Nonlinear Dynamics, vol. 67, no. 2, pp. 1635-1646, 2012.

[11] W. Wu, W. J. Zhou, and T. P. Chen, "Cluster synchronization of linearly coupled complex networks under pinning control," IEEE Transactions on Circuits and Systems I, vol. 56, no. 4, pp. 829-839, 2009.

[12] K. H. Wang, X. C. Fu, and K. Z. Li, "Cluster synchronization in community networks with nonidentical nodes," Chaos, vol. 19, no. 2, Article ID 023106, 10 pages, 2009.

[13] J. D. Cao and L. L. Li, "Cluster synchronization in an array of hybrid coupled neural networks with delay," Neural Networks Letter, vol. 47, no. 11, pp. 2395-2405, 2011.

[14] W. B. Zhang, Y. Tang, J. A. Fang, and W. Zhu, "Exponential cluster synchronization of impulsive delayed genetic oscillators with external disturbances," Chaos, vol. 21, no. 4, Article ID 043137, 2011.

[15] J. Q. Lu and J. D. Cao, "Adaptive synchronization of uncertain dynamical networks with delayed coupling," Nonlinear Dynamics, vol. 53, no. 1-2, pp. 107-115, 2008.

[16] W. L. He and J. D. Cao, "Adaptive synchronization of a class of chaotic neural networks with known or unknown parameters," Physics Letters A, vol. 372, no. 4, pp. 408-416, 2008.

[17] Y. H. Sun and J. D. Cao, "Adaptive lag synchronization of unknown chaotic delayed neural networks with noise perturbation," Physics Letters A, vol. 364, no. 3-4, pp. 277-285, 2007.

[18] W. W Yu and J. D. Cao, "Adaptive synchronization and lag synchronization of uncertain dynamical system with time delay based on parameter identification," Physica A, vol. 375, no. 2, pp. 467-482, 2007.

[19] J. Q. Lu and J. D. Cao, "Adaptive synchronization in tree-like dynamical networks," Nonlinear Analysis: Real World Applications, vol. 8, no. 4, pp. 1252-1260, 2007.

[20] Y. Y. Wu, C. P. Li, A. L. Yang, L. J. Song, and Y. J. Wu, “Pinning adaptive anti-synchronization between two general complex dynamical networks with non-delayed and delayed coupling," Applied Mathematics and Computation, vol. 218, no. 14, pp. 7445-7452, 2012.

[21] D. G. Xu and Z. F. Su, "Synchronization criterions and pinning control of general complex networks with time delay," Applied Mathematics and Computation, vol. 215, no. 4, pp. 1593-1608, 2009.

[22] K. Lou, B. T. Cui, and X. J. Zhang, "Adaptive synchronization of two complex networks with delayed and non-delayed coupling," Arabian Journal of Mathematics, vol. 1, no. 2, pp. 219-226, 2012.

[23] X. J. Wu and H. T. Lu, "Exponential synchronization of weighted general delay coupled and non-delay coupled dynamical networks," Computers and Mathematics with Applications, vol. 60, no. 8, pp. 2476-2487, 2010.

[24] W. L. Lu and T. P. Chen, "New approach to synchronization analysis of linearly coupled ordinary differential systems," Physica D, vol. 213, no. 2, pp. 214-230, 2006.

[25] Y. S. Moon, P. Park, W. H. Kwon, and Y. S. Lee, "Delaydependent robust stabilization of uncertain state-delayed systems," International Journal of Control, vol. 74, no. 14, pp. 14471455, 2001. 


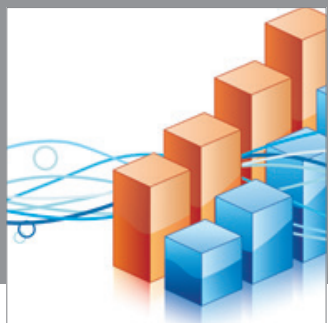

Advances in

Operations Research

mansans

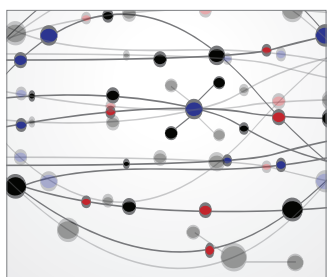

The Scientific World Journal
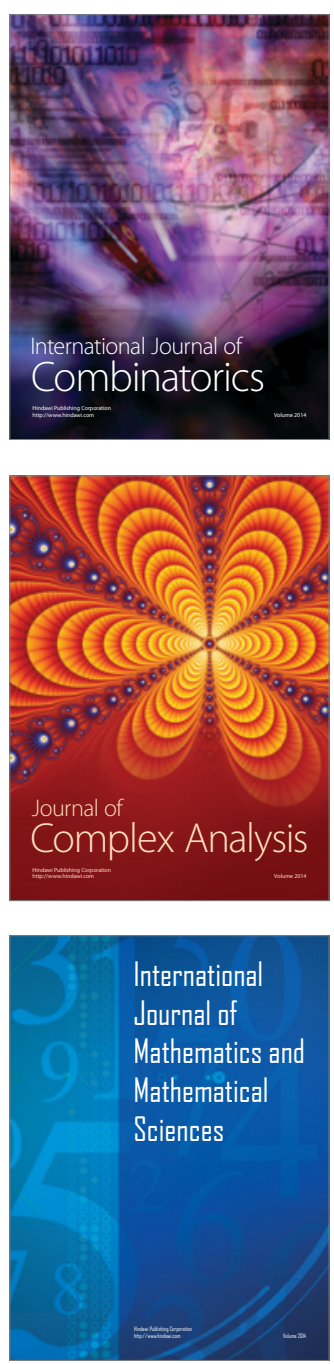
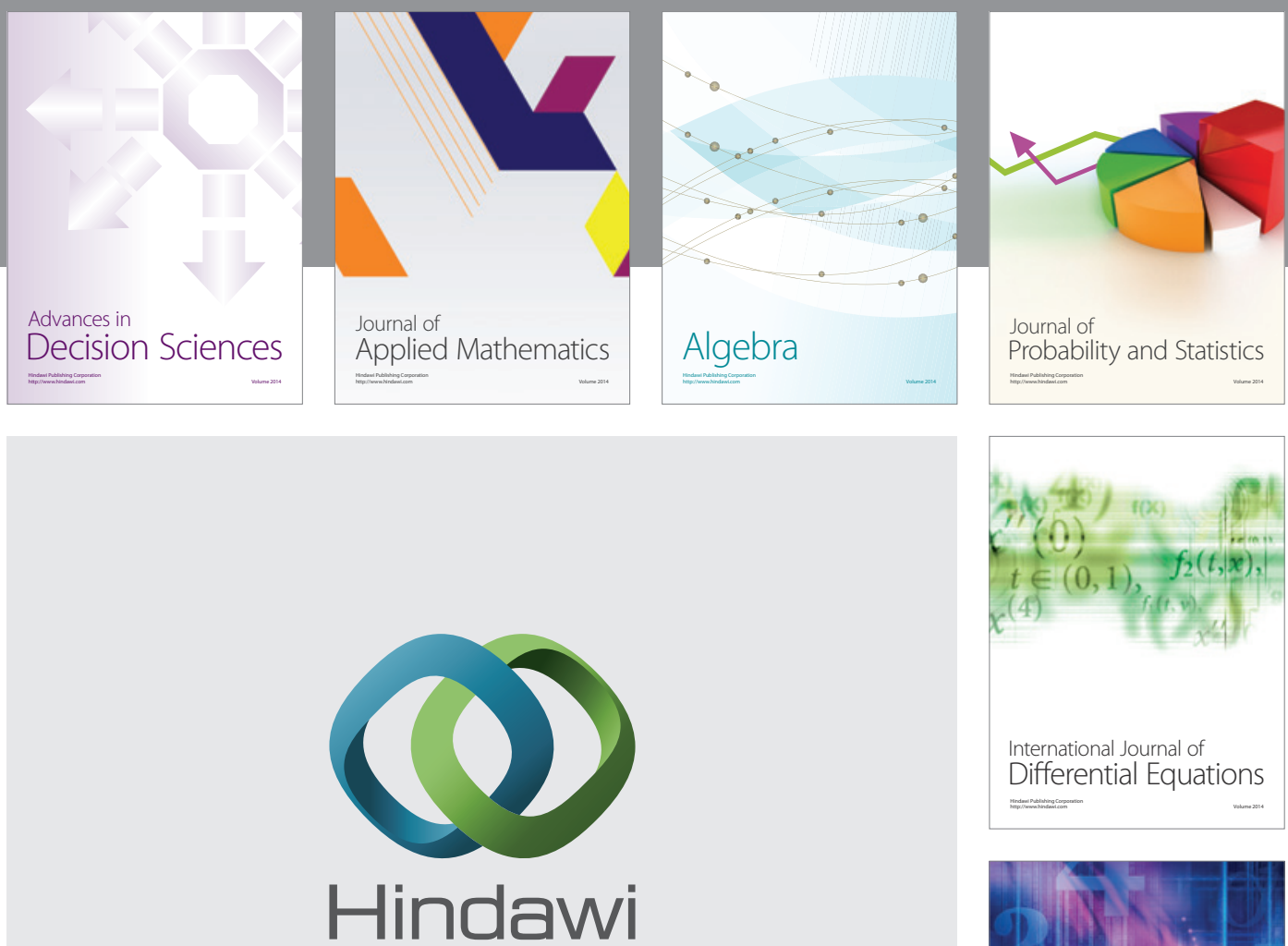

Submit your manuscripts at http://www.hindawi.com
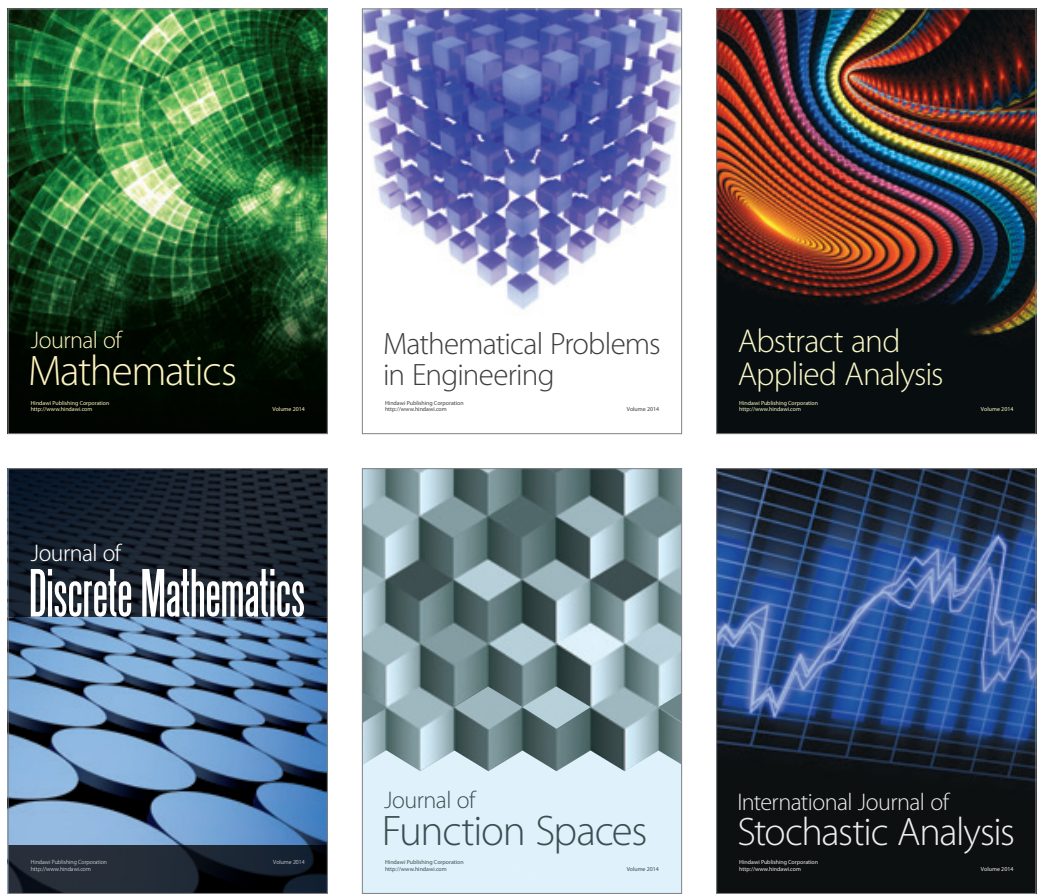

Journal of

Function Spaces

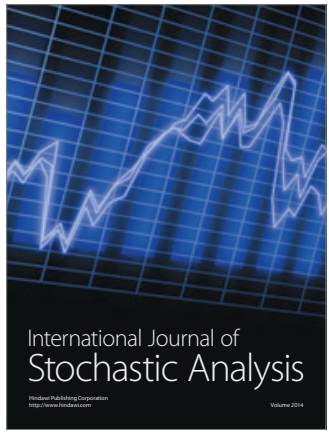

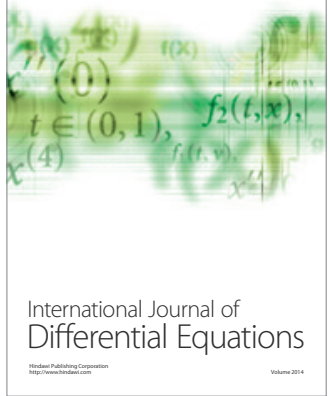
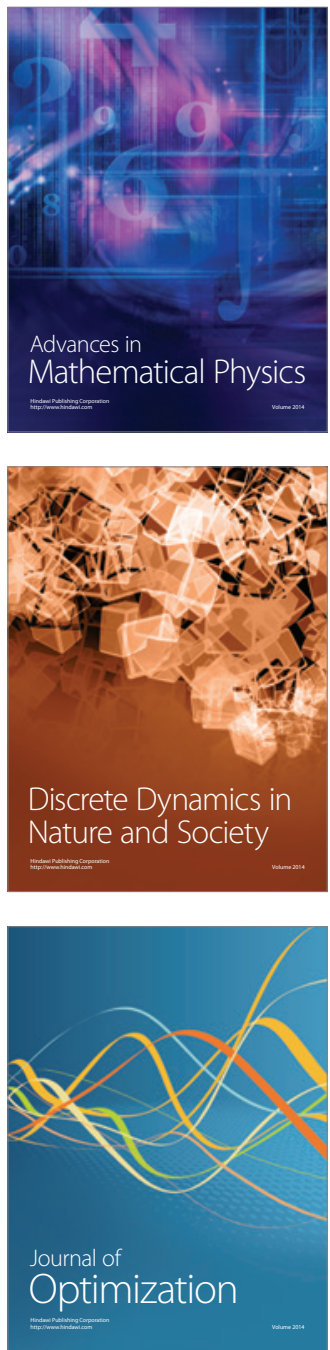